Thorax (1956), 11, 209.

\title{
MULTIPLE SPUTUM CULTURES IN THE ASSESSMENT OF PULMONARY TUBERCULOSIS
}

\author{
BY \\ D. R. HAY \\ From Sully Hospital, Glamorgan
}

(RECEIVED FOR PUBLICATION SEPTEMBER 15, 1955)

The presence or absence of tubercle bacilli in the sputum is of considerable importance in the assessment of pulmonary tuberculosis and frequently determines whether treatment is undertaken.

At Sully Hospital it has been the practice in recent years to examine a relatively large number of sputum or gastric lavage specimens collected on successive days following the admission of a patient. The majority of the patients have received treatment elsewhere and have been referred to Sully Hospital for surgery. A survey has now been made to determine the value of these extensive laboratory investigations, and it is felt that the results are of some interest.

\section{The Present Investigation}

The records of 330 patients were studied retrospectively, including all the present in-patients and 141 unselected patients who had been discharged in the previous 18 months. Sputum or gastric washings were collected on successive days following admission, and cultured on Löwenstein-Jensen media for six weeks. Direct microscopy of concentrated sputum was also done in many cases, but not routinely. The majority of patients were not on antibacterial treatment at the time of investigation.

\section{RESULTS}

Table I records the total number of cultures performed on the 330 patients. One hundred and sixty-eight patients, or $50.9 \%$ of the series, were sputum positive on one or more culture examinations.

Table II indicates the number of cultures which were necessary before a positive resulted. It is clear that $70 \%$ of the positive results followed the first sputum culture, while $10 \%$ of the group required more than 11 and up to 30 examinations before a positive result was received. Only $3.5 \%$ were positive after more than 15 cultures.
TABLE I

TOTAL NUMBER OF CULTURES PERFORMED

\begin{tabular}{c|c}
\hline No. of Cultures & Patients \\
\hline $1-5$ & 36 \\
$6-10$ & 48 \\
$11-15$ & 82 \\
$16-20$ & 99 \\
$21-25$ & 47 \\
$26-30$ & 8 \\
$31-35$ & 7 \\
$36-$ & 3 \\
\hline Total & 330 \\
\hline
\end{tabular}

TABLE II

NUMBER OF CULTURES PERFORMED BEFORE FIRST POSITIVE RECEIVED

\begin{tabular}{c|c}
\hline No. of Cultures & Patients \\
\hline 1 & 118 \\
$2-5$ & 24 \\
$6-10$ & 10 \\
$11-15$ & 10 \\
$16-20$ & 3 \\
$21-25$ & 1 \\
$26-30$ & 2 \\
\hline Total & 168 \\
\hline
\end{tabular}

The number of positive cultures received from the positive group is shown in Table III, as this gives some indication of the laboratory work involved in this investigation. Thus, $41 \%$ of those who proved sputum positive as a result of the first culture subsequently had more than 11 positive cultures returned, which suggests much unnecessary laboratory work.

TABLE III

TOTAL NUMBER OF POSITIVE CULTURES FROM POSITIVE CASES

\begin{tabular}{c|c}
\hline No. of Positive Cultures & Patients \\
\hline 1 & 25 \\
$2-5$ & 63 \\
$6-10$ & 28 \\
$11-15$ & 35 \\
$16-20$ & 11 \\
$21-25$ & 4 \\
$26-30$ & 2 \\
\hline Total & 168 \\
\hline
\end{tabular}


Direct microscopy of concentrated sputum was performed on 195 patients, of whom 117 were in the culture positive group. Forty-seven patients, or $40 \%$ of those tested, were positive on direct as well as culture examination. Seventy cases, or $60 \%$ of those tested, were negative on one or more direct examinations, but positive on culture.

It was not planned to correlate the bacteriological state with the radiological picture, but $35 \%$ of the 230 patients showing no radiological evidence of cavitation were sputum positive. When cavitation was present on admission, $82 \%$ were positive.

Finally, it is worth noting that $34 \%$ of patients who were presumed to be sputum negative in the six months before their admission to Sully Hospital were found to be sputum positive after more exhaustive sputum investigation there.

\section{Discussion}

The original object of this survey was to assess the value of repeated sputum cultures in the investigation of tuberculous patients. Those studied undoubtedly represented a selected series, and the results are limited in their application. However, it is fair to say that the problems which such a group presents are widely encountered today in the management of pulmonary tuberculosis.

The most important conclusion was that, although the majority of positive patients will be revealed by five cultures, approximately $10 \%$ of cases will be missed if the search is not continued beyond 10 cultures. The yield of positive results after 15 cultures is very small, and for routine purposes does not justify the laboratory work involved. It may be argued that a patient with 10 consecutive negative cultures can be regarded as sputum negative. At Sully Hospital, however, a decision to operate has not infrequently followed the appearance of a positive culture after more than 10 previous negative results when there was no other evidence of activity of the lesion.
Direct microscopy of sputum was less extensively used in this series of patients, although it is clearly of considerable importance in the investigation of pulmonary tuberculosis, particularly in acute cases. On the other hand, one must deplore the tendency to regard "three negative directs" as an indication of the bacterial status of a patient, and insist on a full culture examination, which, as Cruickshank says (1952), " must not be regarded, as it sometimes is, as an unnecessary (and annoying) bacteriological refinement."

\section{SUMMARY}

In a series of 330 chronic tuberculous patients, $\bigcirc$ 168 were found to be positive on repeated culture examination of sputum or gastric washings.

One hundred and seventeen patients (or 70\%) were positive as a result of the first culture examination.

Sixteen patients (or $10 \%$ ) were not found to be positive until more than 10 and up to 30 cultures had been performed. Only $3.5 \%$ were positive after more than 15 cultures.

Eighty-two per cent. of patients with persistent cavitation were sputum positive, and $35 \%$ of those with no radiological evidence of cavitation were positive.

It is concluded that a series of 15 consecutive cultures of sputum or gastric washings will in most cases confirm whether a patient is sputum positive or negative.

I wish to acknowledge the assistance of Dr. J. Marks, of the Central Tuberculosis Laboratory, Cardiff, where most of the laboratory work was carried out. I also thank Dr. H. M. Foreman, Physician Superintendent of Sully Hospital, for his permission to publish this report, and Dr. R. M. E. Seal, Consultant Pathologist, for his assistance.

\section{ReFERENCE}

Cruickshank, D. B. (1952). In Modern Practice in Tuberculosis. Vol. 1, p. 53, edited by T. Holmes Sellors and J. L. Livingstone. Butterworth, London. 